\title{
Teilzeitmodelle in der Anästhesie Beispiel einer Anästhesieabteilung eines deutschen Universitätsklinikums
}

\author{
Maike Höltje* • W. Alexander Osthaus* • Wolfgang Koppert \\ * geteilte Erstautorenschaft
}

Teilzeitmodelle werden aus unterschiedlichen Gründen von vielen Seiten zunehmend gefordert. Dennoch sucht man in der deutschsprachigen Literatur vergebens nach konkreten Teilzeitmodellen, insbesondere in der Medizin. Im folgenden Artikel wird erstmalig - nach Betrachtung der Hintergründe und Rahmenbedingungen - ein seit 2 Jahren existierendes Teilzeitmodell einer Anästhesieabteilung an einem Universitätsklinikum in Deutschland vorgestellt. In diesem Teilzeitmodell werden sowohl die Bedürfnisse junger Familien berücksichtigt als auch die Wünsche der älteren Mitarbeiter implementiert.

\section{Problem Ärztemangel}

Topthema Seit Jahren findet man kaum noch eine Ausgabe des Deutschen Ärzteblatts, in der nicht in irgendeiner Weise von Problemen im Zusammenhang mit dem vorhandenen oder zu erwartenden Ärztemangel in Deutschland berichtet wird. Auch die fachspezifischen deutschen Zeitschriften räumen diesem Thema regelmäßig Platz ein. Ebenso widmen sich die unterschiedlichen Berufsverbände und Fachgesellschaften immer häufiger diesem Problem - sei es in berufspolitischen Sitzungen der Fachtagungen oder in der Verbandsarbeit. Politik, deutsche Krankenhausgesellschaft, Bundesärztekammer und Kassenärztliche Bundesvereinigung ergänzen die Institutionen, für die der Ärzte- und Fachkräfte- mangel im deutschen Gesundheitswesen ein Topthema geworden ist.

Im Folgenden wird dargestellt, welche Rahmenbedingungen neben dem Fachkräftemangel dazu geführt haben, dass Teilzeitmodelle heute vermehrt angeboten werden. Anhand von Zahlen aus der Medizinischen Hochschule Hannover (MHH) wird anschließend beispielhaft aufgezeigt, wie ein Teilzeitkonzept für eine anästhesiologische Klinik aussehen kann.

Kontroverse Meinungen Trotz der großen Einigkeit über die Brisanz des Themas gibt es durchaus unterschiedliche Perspektiven. Zum einen wird postuliert, dass es kein Nachwuchsproblem gibt: Die ausscheidenden Ärzte würden durch die neu ausgebildeten ersetzt werden; die

Tab. 1 Daten modfiziert nach [13].

\section{Charakteristika und Werte der Generation Y}

\begin{tabular}{|l|l|}
\hline Jahrgang & ab 1981 \\
\hline Typ & hohes Selbstbewusstsein, nicht kritikfähig \\
\hline Technik & Web 2.0, Handy, „instant messenger“, Laptop \\
\hline Denkweise & pragmatisch, kooperativ, Netzwerke \\
\hline Verhältnis zur Arbeit & $\begin{array}{l}\text { Hierarchien werden abgelehnt; hohes Anforderungsprofil an } \\
\text { Arbeitsplatz: flexibel und ergebnisorientiert, kein Absitzen } \\
\text { von Arbeitszeit, Überstunden müssen sehr gut begründet } \\
\text { werden; wechselt eher den Job, als sich anzupassen }\end{array}$ \\
\hline akadem. Ausrichtung & Bedeutung akademischer Titel nimmt weiter ab \\
\hline Wert der Familie & $\begin{array}{l}\text { Familienbild definiert sich neu: Neuentdeckung konservativer } \\
\text { Werte, Familie genießt höchste Priorität }\end{array}$ \\
\hline Motivation & optimale Ausbildung, gute Supervision, optimale Führung \\
\hline
\end{tabular}

Zuwanderung ausländischer Ärzte - gerade auch nach der Einführung der Blauen Karte EU - sei größer als die Abwanderung aus Deutschland. Die insgesamt attraktiven Arbeitsbedingungen, höheren Einkommen und eine adäquate Beschäftigung bewirkten beruflichen Erfolg und damit einen positiven Wanderungssaldo nach Deutschland [1].

Demgegenüber stehen Prognosen, die bereits 2015 mit einem Fachkräftemangel von 175000 Ärzten und Pflegekräften rechnen [2]. In einer Studie des Deutschen Krankenhausinstituts im Auftrag der Deutschen Krankenhausgesellschaft wird erwartet, dass im Jahr 201937400 Ärzte fehlen werden [3]. Der Andrang auf die Studienplätze ist zwar nach wie vor groß. Die Zahl der ausgebildeten Mediziner, die nach dem Studium keiner kurativen Tätigkeit nachgehen, liegt aber $>10 \%$ und auch die Quote der Studienabbrecher ist hoch, wenn hier auch keine einheitlichen Zahlen vorliegen. Der Kampf um die besten Köpfe hat längst begonnen [4, 5], was u.a. an der zunehmenden Zahl von Jobmessen für Medizinstudenten abzulesen ist.

Junge Arztgeneration Einige Arbeitgeber haben diese Trends mittlerweile realisiert. Doch was sind die Bedürfnisse, Wünsche und Motivatoren der jungen Arztgeneration? Was wird von potenziellen Arbeitgebern erwartet? Auch hier hat es in den letzten Jahren gravierende Veränderungen gegeben ( $\odot$ Tab. 1 ). Je nach Lebenssituation wünschen sich Männer wie Frauen eine Voll- oder Teilzeitbeschäftigung.

Dennoch werden wohl auch in Zukunft mehr Frauen als Männer eine Tätigkeit in Teilzeit anstreben. Wenn der Nachwuchs da ist, will mehr als die Hälfte der Ärztinnen in Teilzeit arbeiten [6]. Der Umstand, dass über 2 Drittel der Studienabgänger weiblich sind, macht deutlich, wie wichtig dieses Thema auch künftig sein wird.

Teilzeitquote Aktuelle Zahlen belegen, dass die Zahl der in Deutschland tätigen Ärzte so hoch ist wie nie zuvor. Anderer- 


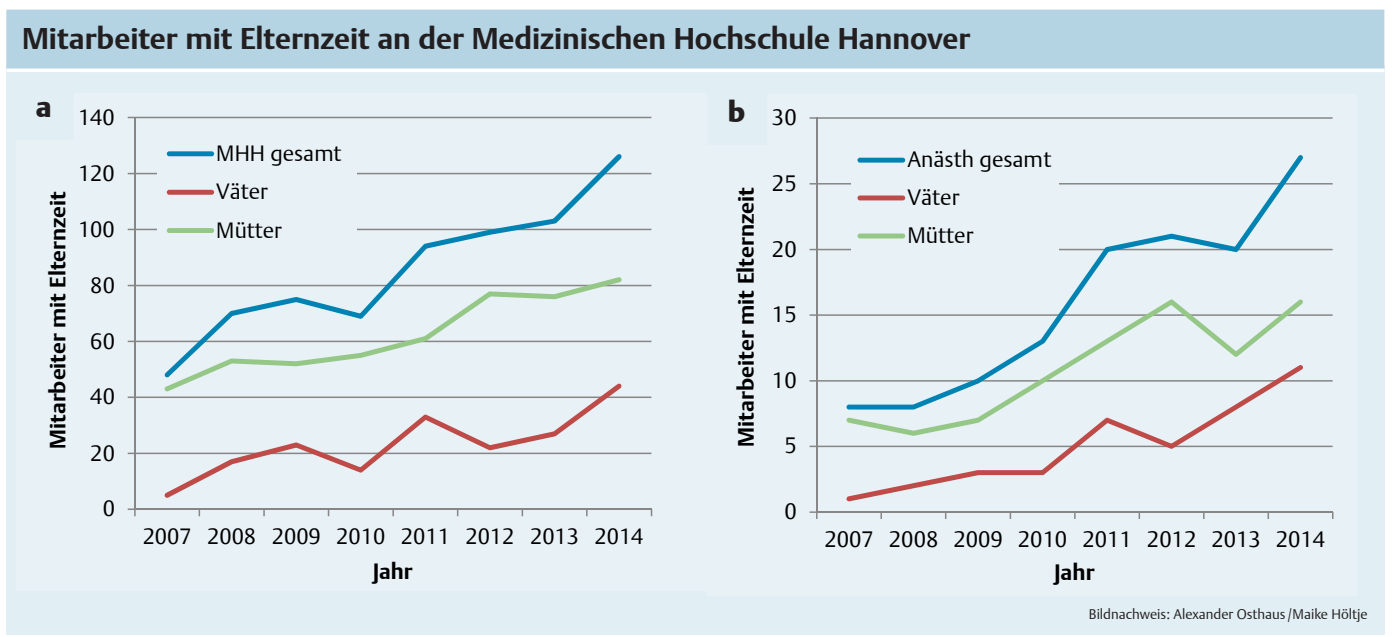

Abb. 1 a Medizinische Hochschule Hannover (MHH) gesamt; b Abteilung für Anästhesiologie. seits steigt auch der Bedarf an Ärzten, da die immer älter werdende Bevölkerung häufigerer Arztkonsultationen bedarf und eine immer größere Anzahl von Ärzten in Teilzeit arbeitet [7]. Nach Zahlen des Statistischen Bundesamts ist der Anteil der in Teilzeit tätigen Ärzte von 2001-2011 um ca. 75\% auf 54000 gestiegen. Ebenso nimmt die Feminisierung des ärztlichen Berufs weiter zu: Der Anteil der Ärztinnen liegt mittlerweile bei $45 \%$ (vor 18 Jahren waren es noch knapp 36\%). Dieser Trend wird sich voraussichtlich vorerst weiter fortsetzen. Hierdurch steigen sowohl die Quote an Teilzeitbeschäftigung als auch der elternzeitbedingte vorübergehende Ausfall von Arbeitskräften weiter an. Somit besteht kein Widerspruch in der numerischen Zunahme von Ärzten auf der einen Seite und dem zunehmenden Ärztemangel auf der anderen Seite $[3,8]$.

Situation in der Anästhesiologie Neben den allgemeinen Veränderungen in der Medizin gibt es auch fachspezifische Besonderheiten. Das Fach Anästhesiologie scheint im Vergleich mit anderen Fächern noch nicht so stark vom Ärztemangel betroffen zu sein. In der Anästhesiologie werden derzeit noch mehr Fachärzte ausgebildet, als für den Ersatz des altersbedingten Ausscheidens notwendig wären $[9,10]$.

Trotzdem klagen selbst an deutschen Universitätsklinika knapp 40\% der Anästhesieabteilungen über eine unzureichende Stellenausstattung; 80\% der Universitätsklinika gaben 2011 an, in den letzten 12 Monaten unbesetzte Stellen gehabt zu haben. Im Mittel konnten 2,8 Stellen pro Monat nicht besetzt werden [9]. Diese Situation ist in kleineren Häusern regelhaft noch problematischer. Das spiegelt sich auch in einer Umfrage des Berufsverbandes Deutscher Anästhesisten 2009 wieder. Hier gaben über die Hälfte der befragten Chefärzte an, bereits auf Honorarärzte zurückzugreifen. Bei beständig ansteigenden Eingriffs- und OP-Zahlen ist davon auszugehen, dass der Bedarf an Anästhesisten in den kommenden Jahren noch weiter steigen wird [5].

Charakteristika des Fachs Eine Ursache für offene Stellen in der Anästhesiologie ist auf die Veränderungen im Arbeitsalltag zurückzuführen. Die letzten Jahre waren im Fach Anästhesiologie geprägt vom DRG-Zeitalter mit einer erheblichen Arbeitsverdichtung und zunehmenden kennzahlenbasierten und -gesteuerten Arbeitsprozessen. Die persönliche Einflussnahme auf die eigene Arbeit, die durch das systemimmanente Problem der (gefühlten) Fremdbestimmung durch die Kollegen der operativen Abteilungen oft sowieso schon als gering eingeschätzt wird, wird von der jungen Arztgeneration teilweise noch kritischer gesehen.

Zusätzlich haben die veränderten Arbeitsprozesse (z.B. Entkopplung des Prämedikationsgesprächs) für viele Anästhesisten nur noch punktuelle Patientenkontakte zur Folge, die das Verständnis für das eigentliche Arztsein zunehmend vermissen lassen. Das fördert weder die ArztPatienten-Beziehung noch nützt es dem Ansehen des Faches; es erleichtert auf der anderen Seite aber Teilzeitmodelle.

Ist-Situation Heute schon nehmen die Bewerber ihre potenziellen Arbeitgeber auch bezüglich der Frage „Vereinbarkeit von Familie und Beruf" genauestens unter die Lupe. Eine Umfrage unter Medizinstudenten 2009 ergab, dass vielfältige berufliche Perspektiven und ein ausge- wogenes Verhältnis von Beruf und Freizeit/Familie besonders wichtig sind bei der späteren Berufswahl [4]. Beides sahen die Studenten für das Fach Anästhesiologie schlechter verwirklicht als sie es sich wünschten.

Auch von den berufstätigen Anästhesisten wird der Konflikt zwischen Berufs- und Privatleben überdurchschnittlich hoch bewertet [11]. Seit der Einführung des Elterngeldes 2007 ist bundesweit der Anteil von Vätern, die Elternzeit nehmen, stetig gestiegen (von 20,3\% in 2008 auf $28,8 \%$ in 2012 laut Statistischem Bundesamt). Gleiches trifft sowohl auf die MHH als Ganzes als auch auf die Abteilung für Anästhesiologie im Speziellen zu (o Abb. 1)

Darüber hinaus steigt auch der Anteil der Väter in Teilzeitarbeit ständig weiter [12]. Elternzeit und Teilzeittätigkeit für Männer und Frauen scheinen salonfähig $\mathrm{zu}$ werden - zumindest in der Medizin. Dabei geht es nicht nur um eine reduzierte Wochenstundenzahl, sondern auch um die Möglichkeit längerer „Auszeiten“, z. B. in Form von Elternzeit oder Sabbaticals. Schreitet diese Entwicklung fort, wird Teilzeitarbeit künftig nicht mehr die Ausnahme, sondern die Regel sein [4].

\section{Lösungsansätze \\ $\nabla$}

Was ist machbar? Jede Abteilung sollte sich ab sofort nicht nur damit auseinandersetzen, ob eine Teilzeittätigkeit möglich ist, sondern auch, wie. Ziel muss es sein, die Interessen bzw. Möglichkeiten des Krankenhauses mit den Interessen bzw. Wünschen des Arbeitnehmers abzugleichen und eine möglichst große Schnittmenge zu bilden. Das Mögliche hängt dabei sehr stark von der individuel- 


\begin{tabular}{|l|c|c|c|c|}
\hline \multicolumn{2}{|c|}{ Kinderbetreuungsplätze an der MHH } & & \\
& $\begin{array}{c}\text { MHH } \\
\text { (seit 1970) }\end{array}$ & $\begin{array}{c}\text { Elterninitiative } \\
\text { (seit 1991) }\end{array}$ & $\begin{array}{c}\text { JUH } \\
\text { (seit 2010) }\end{array}$ & $\Sigma$ \\
\hline Krippe (<3 Jahre) & 60 & 30 & 65 & 155 \\
\hline Kindergarten (3-6 Jahre) & 82 & 50 & 65 & 197 \\
\hline Hort (6-14 Jahre) & 44 & - & - & 44 \\
\hline Anzahl Plätze insgesamt & 186 & 80 & 130 & 396 \\
\hline
\end{tabular}

Tab. 2 Art und Anzahl der Kinderbetreuungsplätze an der Medizinischen Hochschule Hannover (MMH), aufgelistet nach den verschiedenen Trägern. JUH= Johanniter Unfall Hilfe e. V.

len Arbeitsorganisation des Faches und der Kreativität und Flexibilität der Abteilung ab. Voraussetzung ist aber ein entsprechend umfassendes Kinderbetreuungsangebot. Hier kann das Krankenhaus als Träger einer Betriebs-Kindertagesstätte (Kita) die nötigen Rahmenbedingungen schaffen.

Kinderbetreuung Eine wohnort- oder arbeitsplatznahe Kinderbetreuung zu finden, ist für viele Beschäftigte schwierig auch nach Einführung des Anspruchs auf einen Kindergartenplatz. Häufig passen die Anfangs- bzw. Endzeiten nicht zu den Arbeitszeiten in einem Krankenhaus. Die Kinderbetreuungszeiten müssten z. B. für das Fach Anästhesiologie optimalerweise vor Beginn der OP-Laufzeiten starten und nach diesen enden.

Hier könnte das Krankenhaus als Arbeitgeber eine große Verantwortung übernehmen und eine ausreichende Anzahl an Kinderbetreuungsplätzen mit entsprechenden Betreuungszeiten zur Verfügung stellen. Idealerweise sollte es auch eine Notfall-Kinderbetreuung geben, die z.B. einspringen kann, wenn die Tagesmutter mal krank ist. Eine verlässliche Kinderbetreuung ist eine Grundvoraussetzung dafür, dass sich Eltern während ihrer Arbeitszeit ganz ihrer beruflichen Tätigkeit widmen können.

Kinderbetreuung an der $\mathrm{MHH}$ Die MHH hat 1970, 5 Jahre nach ihrer Gründung, ihre erste Betriebs-Kita eröffnet. Im Laufe der letzten 45 Jahre sind 2 weitere Ganztages-Kitas dazugekommen: eine Elterninitiative und eine Kita in Trägerschaft der Johanniter Unfall Hilfe e. V. Die Öffnungszeiten orientieren sich an den Arbeitszeiten der Mitarbeiter mit einer Kernöffnungszeit sowie Früh- und Spätdiensten. Mit allen 3 Kitas zusammen stehen an der MHH für 7607 Vollkräfte (VK) und 3321 Studenten (2013) insgesamt knapp 400 ganztägige Kinderbetreuungsplätze zur Verfügung ( $\bullet$ Tab. 2). Neben den Kitas bietet die MHH noch eine gesonderte Kinderbetreuung während Prüfungen, Kongressen und Fortbildungen sowie eine kostenlose Notfall-Kinderbetreuung in Kooperation mit einem externen Dienstleister an. Gerade die Notfall-Kinderbetreuung ermöglicht z. B. bei kurzfristiger Erkrankung der Tagesmutter oder Schließung des Kindergartens eine Lösung. Erfolgt der Anruf bis $18 \mathrm{Uhr}$ am Vortag, wird die Kinderbetreuung sogar

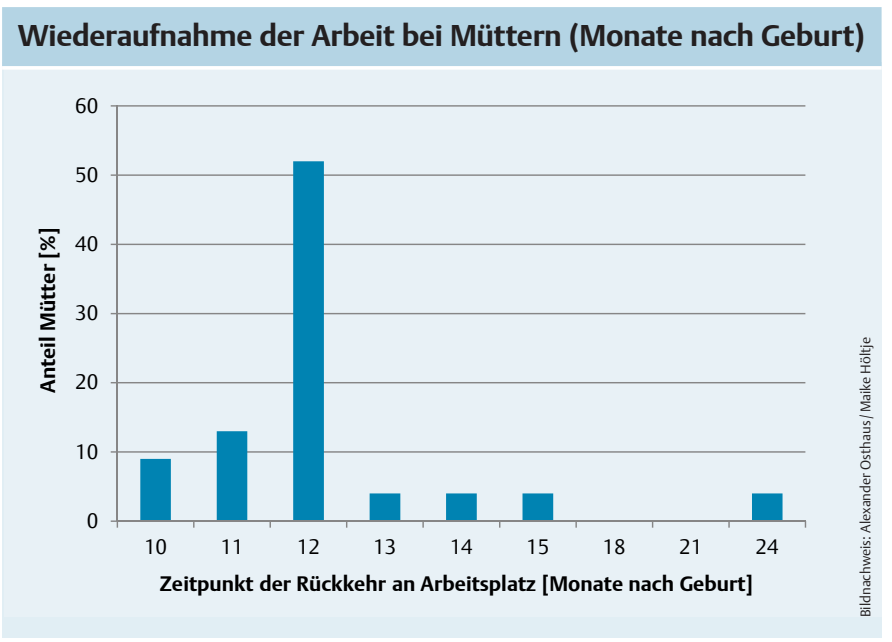

Abb. 2 Zeitpunkt der Wiederaufnahme der Arbeit von Müttern nach der Geburt in der Abteilung für Anästhesiologie der $\mathrm{MHH}$ im Zeitraum von 2011-2014. garantiert. 2013 wurden an 149 Tagen zusätzliche Kinderbetreuungen ermöglicht. Außerdem haben 79 Familien mit 104 Kindern insgesamt 322 Notfall-Kinderbetreuungstage in Anspruch genommen.

Flexible Lösungen Trotz der vielen Möglichkeiten ist die Nachfrage weiterhin deutlich größer als das Angebot. Viele Familien müssen deshalb auf Angebote außerhalb der Betriebs-Kita zurückgreifen. Die Betreuungszeiten in diesen externen Einrichtungen sind jedoch häufig nicht kompatibel mit dem üblichen Arbeitsbeginn in einer Klinik. Um dann Mitarbeitern nach z.B. Schwangerschaft, Geburt und Elternzeit überhaupt einen Wiedereinstieg an ihrem alten Arbeitsplatz zu ermöglichen, müssen Wege gefunden werden, um die Arbeitszeiten entsprechend an die Kinderbetreuungszeiten anzupassen. Häufig sind hiervon sowohl der Arbeitsbeginn als auch das Arbeitsende betroffen. Dies ist für viele Abteilungen eine große Herausforderung.

\section{Teilzeitkonzept \\ $\nabla$}

Situation an der MHH Auch in unserer Abteilung wurde immer häufiger der Wunsch nach einer Tätigkeit in Teilzeit geäußert. Viele Mütter möchten ca. 1 Jahr nach der Kindesgeburt wieder an ihren Arbeitsplatz zurückkehren ( $\bullet$ Abb. 2). Vor allem ein verspäteter Arbeitsbeginn und ein früheres Arbeitsende wurden von immer mehr Kolleginnen und Kollegen mit Kindern gewünscht. Mit der Zeit wurde es jedoch immer schwieriger, die Bedürfnisse der Mitarbeiter mit den Anforderungen eines reibungslosen OPBetriebs in Einklang zu bringen, da die personalplanerischen Kompensationsmöglichkeiten immer weniger Spielraum zuließen.

Ein Weg Vor 2 Jahren wurde eine Strategie entwickelt, wie die Wünsche möglichst vieler Mitarbeiter berücksichtigt werden können, ohne die Kernaufgaben der Abteilung zu vernachlässigen. Mittlerweile arbeiten in unserer Abteilung 38 von 140 Mitarbeitern in Teilzeit ( $\odot$ Abb. 3), mit einem prozentualen VK-Anteil von 19,5\%. Der Anteil der in Teilzeit tätigen Kollegen ist von $17,3 \%$ Anfang 2008 auf 27,3\% Ende 2014 gestiegen. Der Stellenanteil reicht hier von $0,2-0,9 \mathrm{VK}(\odot$ Abb. 4$)$. Unser aktuelles Teilzeitmodell orientiert sich an den Herausforderungen der Kinderbetreuung in den unterschiedlichen 
Lebensabschnitten der Kinder, berücksichtigt aber auch die Betreuung anderer Angehöriger und den Wunsch weiterer Kollegen, ihre Wochenarbeitszeit zu begrenzen. Weiterhin wird berücksichtigt, ob die zu betreuenden Kinder in einem Haushalt mit 1 erwachsenen Betreuungsperson lebt ( $₫$ Tab. 3 ) oder ob für die Betreuung 2 Personen verantwortlich sind (๑ Tab. 4).

Mit Kindern vom 1.-3. Lebensjahr Mitarbeiter mit Kindern zwischen dem 1.- 3. Lebensjahr können ihre Arbeitszeit flexibel nach ihren Wünschen gestalten. Auch Bereitschaftsdienste brauchen nur an Wochenenden abgeleistet werden, wenn die Betreuung der Kinder unter der Woche sonst nicht gewährleistet ist. Bei dieser Regelung wird davon ausgegangen, dass die Betreuung der Kinder durch einen Lebenspartner oder die Familie/Freunde amWochenendeeinfacherzuorganisieren ist als unter der Woche. Da besonders der späte Arbeitsbeginn eine organisatorische Herausforderung ist, den Kolleginnen andereseits aber ein früher Wiedereinstieg ins Arbeitsleben ermöglicht werden soll, ist dieses Modell jetzt nur noch Kolleginnen vorbehalten, deren Kinder das 4. Lebensjahr noch nicht vollendet haben.

Mit Kindern vom 4.-11. Lebensjahr Im Kindergarten- und Grundschulalter erscheint es zumutbar, die Arbeitszeit in einem gewissen Korridor abzuleisten. Die Kinder sind dann in einem Alter, in dem sie eine Betreuung durch Dritte besser akzeptieren. Allerdings wird Alleinerziehenden auch während dieser Zeit eine flexible Arbeitszeitgestaltung ermöglicht ( $\odot$ Tab. 3). Hier wird davon ausgegangen, dass sie deutlich größere Herausforderungen zu meistern haben als Familien mit 2 erwachsenen Betreuungspersonen. Aus diesem Grund wird alleinerziehenden Eltern mit Kindern ermöglicht, auch in diesem Lebensalter Bereitschaftsdienste nur am Wochenende zu leisten.

Mit Kindern ab dem 11. Lebensjahr Gehen die Kinder in weiterführende Schulen, sind sie in der Regel so selbstständig, dass den Eltern zugemutet werden kann, morgens regulär mit der Arbeit zu beginnen. Auch alleinerziehende Mitarbeiter sollten mind. bis $13 \mathrm{Uhr}$ arbeiten können. In Familien mit 2 Erziehungspersonen sollte es möglich sein, bis $14 \mathrm{Uhr}$ zu arbeiten.

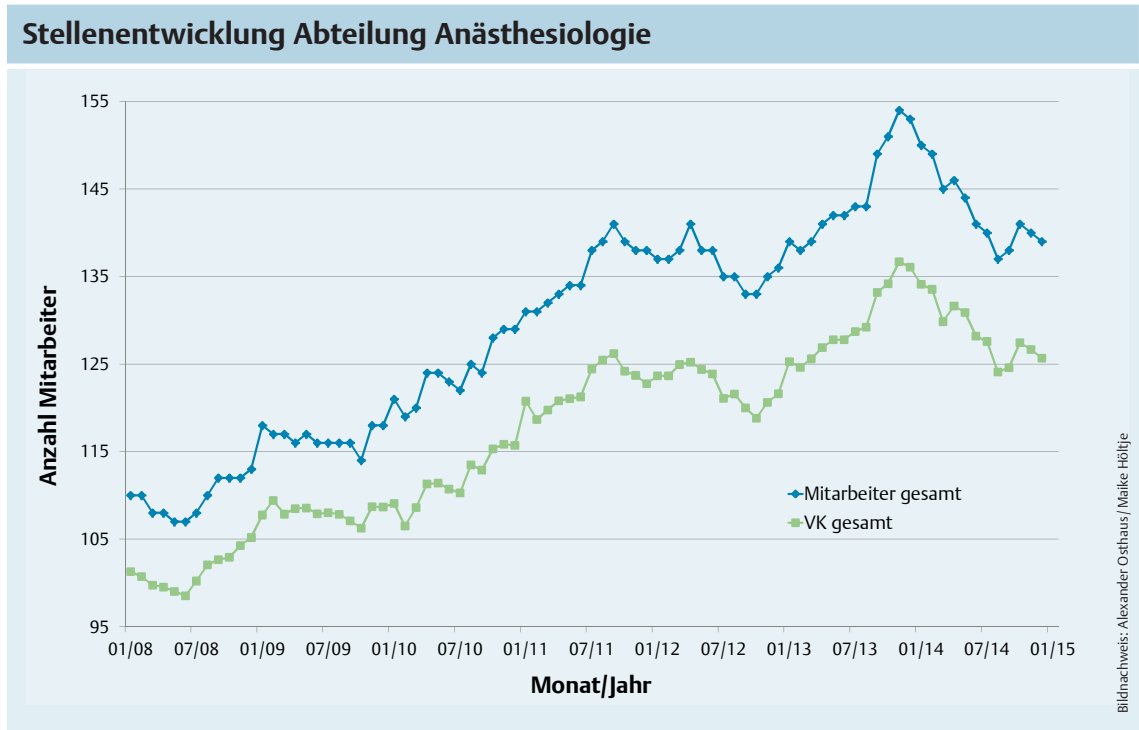

Abb. 3 VK=Vollkräfte.

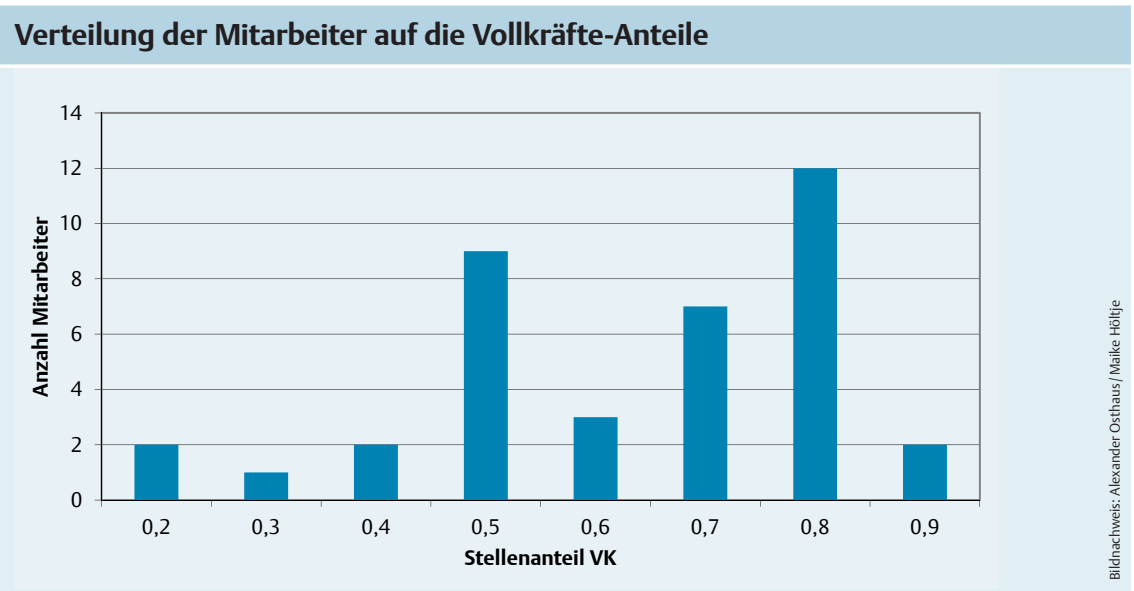

Abb. 4 Zusammenfassung in Bereiche (z. B. 0,5-0,59). VK=Vollkräfte.

\section{Teilzeitmodell A (Familien mit 1 erwachsenen Betreuungsperson)}

\begin{tabular}{|l|l|l|l|}
\hline Alter der Kinder & $\mathbf{1 . - 3 . ~ L J ~}$ & 4.-10. LJ & ab 11. LJ \\
\hline Betreuungseinrichtung & Krippe & $\begin{array}{l}\text { Kindergarten } \\
\text { und Grundschule }\end{array}$ & $\begin{array}{l}\text { weiterführende } \\
\text { Schule }\end{array}$ \\
\hline Arbeitsbeginn & flexibel & flexibel & $\leq 8 \mathrm{Uhr}$ \\
\hline Arbeitsende & flexibel & flexibel & $\geq 13 \mathrm{Uhr}$ \\
\hline tageweise AZ-Reduktion & möglich & möglich & möglich \\
\hline
\end{tabular}

Tab. 3 AZ=Arbeitszeit; $L$ = Lebensjahre.

\begin{tabular}{|l|l|l|l|}
\hline \multicolumn{3}{|l|}{ Teilzeitmodell B (Familien mit $\mathbf{2}$ erwachsenen Betreuungspersonen) } \\
\hline Alter der Kinder & 1.-3. LJ & 4.-10. LJ & ab 11. LJ \\
\hline Betreuungseinrichtung & Krippe & $\begin{array}{l}\text { Kindergarten } \\
\text { und Grundschule }\end{array}$ & $\begin{array}{l}\text { weiterführende } \\
\text { Schule }\end{array}$ \\
\hline Arbeitsbeginn & flexibel & $\leq 8: 30 \mathrm{Uhr}$ & $\leq 8 \mathrm{Uhr}$ \\
\hline Arbeitsende & flexibel & $\geq 12 \mathrm{Uhr}$ & $\geq 14 \mathrm{Uhr}$ \\
\hline tageweise AZ-Reduktion & möglich & möglich & möglich \\
\hline
\end{tabular}

Tab. 4 AZ =Arbeitszeit; LJ=Lebensjahre. 


\begin{tabular}{|l|l|l|l|}
\hline \multicolumn{2}{|l|}{ Teilzeitmodell für Mitarbeiter ohne Kinder bzw. Kinder $>\mathbf{1 8}$ jahre } \\
\hline & $\begin{array}{l}\text { Mitarbeiter } \\
>\mathbf{5 5} \text { Jahre }\end{array}$ & $\begin{array}{l}\text { Mitarbeiter, die pflegebe- } \\
\text { dürft. Angehörige betreuen }\end{array}$ & $\begin{array}{l}\text { Mitarbeiter } \\
\text { ohne Kinder }\end{array}$ \\
\hline BD/RD & auf Wunsch & ja & ja \\
\hline Arbeitsbeginn & regulär & individuelle Regelung & regulär \\
\hline Arbeitsende & regulär & $\begin{array}{l}\text { nach Rücksprache Chefarzt } \\
\text { möglich }\end{array}$ & regulär \\
\hline tageweise AZ-Reduktion & möglich & möglich \\
\hline
\end{tabular}

Tab. $5 \mathrm{BD} / \mathrm{RD}=$ Bereitschaftsdienst $/$ Rufdienst.

Generation 50 plus Jahrelange Bereitschafts- und Schichtdienste sowie die regelmäßige Tätigkeit an Wochenenden und Feiertagen zehren an der Substanz v.a. älterer Kollegen. Für einige kommt in diesem Alter durch die häusliche Pflege von Angehörigen eine weitere Belastung hinzu. Um die Gesundheit dieser Kollegen bestmöglich zu erhalten, bieten wir jedem Mitarbeiter ab 55 Jahren auch ohne ärztliches Attest die Möglichkeit, nicht mehr an Bereitschafts- und Rufdiensten teilnehmen zu müssen ( $\odot$ Tab. 5). Dieses Angebot wird derzeit zu 100\% angenommen.

Mitarbeiter, die aufgrund der Pflege von Angehörigen vermehrt häusliche Verpflichtungen haben, können nach einer individuellen Absprache mit dem Chefarzt für einen begrenzten Zeitraum ihre Arbeitszeit an die Notwendigkeiten des privaten Umfelds anpassen. Dies beinhaltet auch eine untertägige Reduktion der Arbeitszeit. Die tage- bzw. wochenweise Reduktion der Arbeitszeit steht gerade auch den älteren Kollegen offen.

Von den 38 in Teilzeit tätigen Kollegen sind $11>50$ Jahre alt, was einem Anteil von $44 \%$ in dieser Altersgruppe entspricht; in der Gruppe > 55 Jahre sind es 8 Kollegen (57,1\%). Lediglich eine Kollegin in dieser Altersgruppe hat schulpflichtige Kinder. Drei Kollegen gehen einer Teilzeittätigkeit nach, um Angehörige zu Hause betreuen zu können.

Tage- und wochenweise Reduktion Die Variante einer tageweisen Arbeitszeitreduktion stellt für die Personaleinsatz- planung die geringsten Probleme dar. Dieses Modell ist für Familien mit Schulkindern gedacht, kann aber ebenso gut zur Betreuung/Pflege von Angehörigen oder aus anderen rein privaten Gründen genutzt werden. Hier sind alle möglichen Varianten von der 1- bis 4-Tage-Woche denkbar. Gerne wird diese Variante auch mit den Modellen A oder B ( $\odot$ Tab. 3, 4) kombiniert. Auch wöchentliche Wechsel von z.B. 2- und 3-Tage-Woche sind möglich.

Ebenso kann die Arbeitszeit wochenweise reduziert werden. Dies kann starr über das ganze Jahr verlaufen, z.B. bei einer $75 \%$-Stelle 3 Wochen arbeiten - 1 Woche frei oder bei einer $80 \%$ Stelle 4 Wochen arbeiten - 1 Woche frei. Es kann aber auch flexibel mit dem Mitarbeiter besprochen werden, wie die Teilzeitwochen liegen sollen. Hier muss dann nur auf eine möglichst gute Synchronisation der unterschiedlichen Wochenmodelle geachtet werden.

Derzeit nutzen 13 Kollegen die tageweise Arbeitszeitreduktion, während 6 Kollegen lieber wochenweise reduziert haben (o Tab. 6).

Verspäteter Arbeitsbeginn Die größte Herausforderung beim späteren Arbeitsbeginn ist die Sicherstellung des reibungslosen Ablaufs im OP. Zwar bieten große Kliniken auch einzelne klinische Arbeitsplätze, die später als der übliche Arbeitsbeginn starten. Häufig erfordern diese jedoch eine höhere Qualifikation, die zu Beginn des Familienlebens oft noch nicht gegeben ist (Akutschmerzdienst, inter-

Tab. 6 Aktuelle Verteilung der in Teilzeit tätigen Mitarbeiter pro Modell, ohne Mitarbeiter in Elternzeit.

\begin{tabular}{l|l|l|l|l|l|l|l|}
\hline \multicolumn{3}{|l|}{ Mitarbeiter pro Teilzeitmodell } \\
\hline $\begin{array}{l}\text { Arbeitsbeginn - } \\
\text { Arbeitsende }\end{array}$ & $\begin{array}{l}\text { flexibel - } \\
\text { flexibel }\end{array}$ & $\begin{array}{l}\text { regulär - } \\
\geq 12 \text { Uhr }\end{array}$ & $\begin{array}{l}\text { regulär - } \\
\geq 13\end{array}$ Uhr & $\begin{array}{l}\text { regulär - } \\
\geq 14 \text { Uhr }\end{array}$ & $\begin{array}{l}\text { tage- } \\
\text { weise }\end{array}$ & $\begin{array}{l}\text { wochen- } \\
\text { weise }\end{array}$ \\
\hline Frauen $(n=27)$ & 2 & 1 & 1 & 13 & 7 & 3 \\
\hline Männer $(n=11)$ & 1 & 0 & 1 & 0 & 6 & 3 \\
\hline
\end{tabular}

ventionelle, diagnostische Eingriffe). Zusätzlich müssen die Kollegen in der Weiterbildung natürlich auch während der Zeit ihrer reduzierten Tätigkeit ihre Weiterbildung komplettieren können. Daher können sie nicht nur in ausgewählten Bereichen eingesetzt werden (z.B. Prämedikationsambulanz).

Je nach OP-Programm ist es in einzelnen Bereichen möglich, dass die Narkose zunächst vom zuständigen Oberarzt begonnen wird und die Teilzeitkraft den Saal später übernimmt. Sollte dies nicht gehen, wird der zeitgerechte Beginn zunächst durch Kollegen abgedeckt, die z.B. für administrative Aufgaben in der studentischen Lehre/Dienstplangestaltung oder für Lehrveranstaltungen mit späterem Beginn eingeteilt sind. Trotz dieser relativ vielen Disponiermöglichkeiten einer großen Universitätsklinik ist die Anzahl der Kollegen, denen ein verspäteter morgendlicher Beginn ermöglicht werden kann, begrenzt.

Früheres Arbeitsende In vielen OPBereichen gibt es inzwischen festgelegte OP-Betriebszeiten, die sich an der Kernarbeitszeit der Mitarbeiter orientieren bzw. darüber hinausgehen. Es gibt kaum noch OP-Bereiche, in denen ein verlässliches Arbeitsende vor der üblichen Betriebszeit liegt. Damit ist ein verkürzter Arbeitstag für Kollegen nur möglich, wenn sie in ihrem Saal z.B. von einem Spätdienst abgelöst werden. Spätdienste bieten darüber hinaus zusätzlich die Möglichkeit, die übliche OP-Betriebszeit über die Kernarbeitszeit auszudehnen und damit den Funktionsbereich OP effizienter zu nutzen.

In unserer Abteilung wurden insgesamt 6 Spätdienste für den OP-Bereich eingeführt. Vier Mitarbeiter kommen um 11 Uhr und weitere 2 Mitarbeiter um 14 Uhr. Diese Spätdienste lösen während der Phase der Kernarbeitszeit Teilzeitkräfte mit verkürzter Arbeitszeit ab oder Kollegen, die Studentenunterricht übernehmen. Damit entspricht dieser Arbeitsrhythmus funktionell einem Schichtbetrieb, in dem die Kollegen, die aus Gründen der Kinderbetreuung vormittags arbeiten, in den Mittagsstunden von Spätdiensten abgelöst werden.

Begrenzung der Wochenarbeitszeit Ein Konzept, dass Eltern von kleinen Kindern wieder in die Arbeitswelt integrieren soll, lässt fast zeitgleich Rufe nach Konzepten für Familien laut werden, die anderweitige Angehörige betreuen wol- 
len / müssen und dafür entsprechend ihre Arbeitszeit anpassen wollen. In diesem Zusammenhang müssen auch die Mitarbeiter erwähnt werden, welche die durch Bereitschaftsdienste erhöhte Wochenarbeitszeit begrenzen möchten. Für beide Gruppen haben wir zusätzlich flexible Kompensationstage für Bereitschaftsdienste eingeführt. Sie werden nach einem Wunschplan monatlich vergeben und bieten so Arbeitgeber und Arbeitnehmer eine große Flexibilität in der Personaleinsatzplanung. Wird für Überstunden bzw. Bereitschaftsdienste Freizeitausgleich gewährt, kann für den eingesparten Arbeitslohn ab einer gewissen Größenordnung ein neuer Mitarbeiter eingestellt werden. All diese Möglichkeiten sind auch ein wesentlicher Beitrag zur Gesunderhaltung der Arbeitnehmer:

Anmerkung Derzeit lassen die Altersstruktur der Abteilung und die Anzahl der jungen Eltern mit Wunsch nach Teilzeittätigkeit die in diesem Artikel beschriebenen Angebote zu. Uns ist bewusst, dass das Konzept bei Veränderungen der Rahmenbedingungen reevaluiert und ggf. angepasst werden muss.

Fazit Der Wettbewerb um die besten Nachwuchskräfte hat in Anbetracht des zunehmenden Nachwuchsmangels längst begonnen. Es stellt sich daher nicht mehr die Frage ob, sondern welches Teilzeitkonzept man den aktuellen bzw. künftigen Mitarbeitern anbieten kann. Wir favorisieren für Familien ein auf die Bedürfnisse des Mitarbeiters angepasstes und auf das Alter des Kindes bezogenes Stufenkonzept. Eine Begrenzung der Wochenarbeitszeit in Verbindung mit einer Reduktion der Dienstbelastungen ist insbesondere für ältere Arbeitnehmer interessant. In Verbindung mit einer kalenderjährigen Befristung der Teilzeitverträge behält man als Arbeitgeber auch in einer Uniklinik noch die nötige Flexibilität bei gleichzeitiger Vereinfachung der administrativen Tätigkeit. «

\section{Literaturverzeichnis}

1 Demary V, Koppel O. Der Arbeitsmarkt für Humanmediziner und Ärzte in Deutschland - Zuwanderung verhindert Engpässe. IW-Trends - Vierteljahresschrift zur empirischen Wirtschaftsforschung 2013; 40: 1-17

2 Roland Berger Strategy Consultants. Fachkräftemangel im Gesundheitswesen. 2013. Im Internet: http://www.rolandberger.com/media (Stand vom 28.10.2013)

3 Blum K, Löffert S. Ärztemangel im Krankenhaus - Ausmaß, Ursachen, Gegenmaßnahmen. Forschungsgutachten im Auftrag der Deutschen Krankenhausgesellschaft. Düsseldorf: Deutsches Krankenhausinstitut e.V.; 2010

4 Welker A, Baumgart A, Baja J et al. Das Berufsbild des Anästhesisten - Eine qualitative und quantitative Studierendenbefragung zur Attraktivität unseres Fachs. Anästh Intensivmed 2010; 51 : 318-327

5 Schüttler J. Editorial. Anästh Intensivmed 2010; 51: 7-8

6 Kopf S. Arbeitsplatz Krankenhaus: Was die jungen Wilden wollen. Dtsch Arztebl 2014; 111: 2-4

7 Hibbeler B. Ärztemangel: Schluss mit der Schuldfrage. Dtsch Arztebl 2010; 107: A-2307

8 Osterloh F. Ärztestatistik: Mehr Ärztinnen, mehr Angestellte. Dtsch Arztebl 2014; 111: A-672673

9 Kaisers UX, Welker A, Busch T et al. Aktuelle Personalsituation im ärztlichen Dienst der Anästhesiologie an universitären Einrichtungen in Deutschland. Anästh Intensivmed 2011; 52: 708-719

10 Knichwitz G, Wenning M. Gehen Deutschland die Anästhesisten aus? Anästh Intensivmed 2009; 50: 276-282

11 Heinke W, Dunkel P, Brähler E et al. Arbeitszufriedenheit von Anästhesisten in Deutschland. Anästh Intensivmed 2009; 50: 7-19

12 Flintrop J. Ärztemangel: Wenn der Nachwuchs fremdgeht. Dtsch Arztebl 2009; 8: 396

13 Schmidt CE, Möller J, Schmidt K et al. Generation Y. Anästhesist 2011; 60: 517-524

Beitrag online zu finden unter http://dx. doi.org/10.1055/s-0040-100507
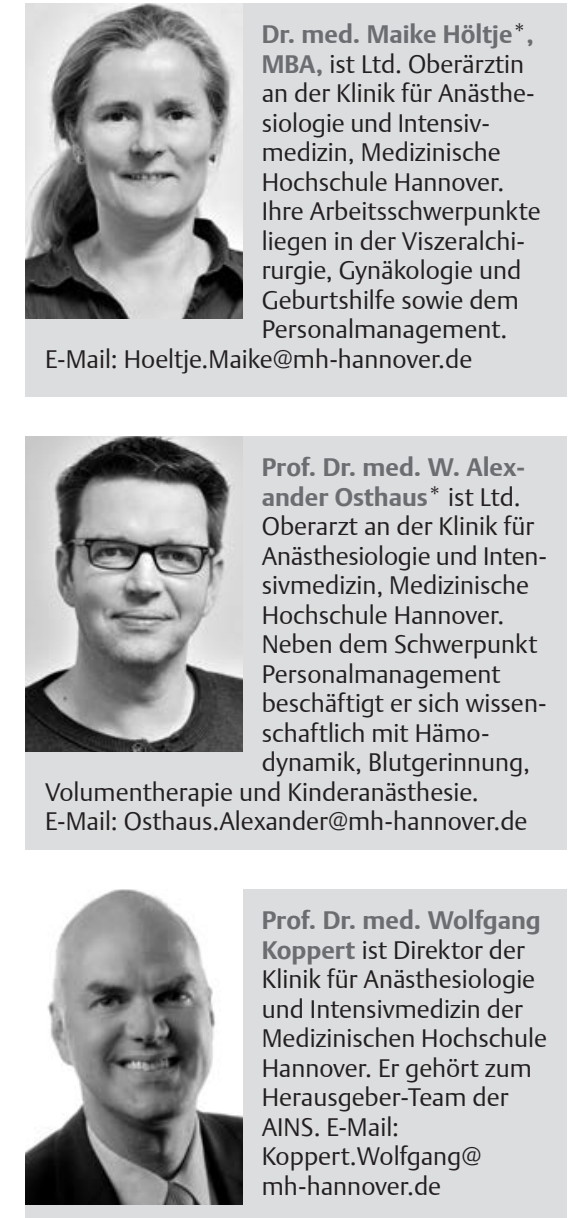

Interessenkonflikt Die Autoren erklären, dass keine Interessenkonflikte vorliegen.

* geteilte Erstautorenschaft 